\title{
Synthesis and antimicrobial activities of novel peptide deformylase inhibitors
}

\author{
Ling Yin, ${ }^{\mathrm{a}}$ Wei-Ren Xu, ${ }^{\mathrm{b}}$ Zhi-Guo Wang, ${ }^{\mathrm{c}}$ Da-Tong Zhang, ${ }^{\mathrm{a}}$ Jiong Jia, ${ }^{\mathrm{a}}$ Yan-Qing Ge, \\ Yan $\mathrm{Li}^{\mathrm{a}}{ }^{\mathrm{a}}$ and Jian-Wu Wang ${ }^{\mathrm{a}}$ *
}

${ }^{a}$ School of Chemistry and Chemical Engineering, Shandong University, 27, Shanda Nanlu, Jinan 250100, People's Republic of China

${ }^{b}$ The Pharmaceutical Research Institute of Tianjin, 308, Anshan Xidao, Nankai District, Tianjin 300193, People's Republic of China

${ }^{c}$ Northwest Institute of Plateau Biology, Chinese Academy of Sciences, 23, Xinning Road, Xining 810001, People's Republic of China

E-mail: jwwang@sdu.edu.cn

\begin{abstract}
A new series of $\mathrm{N}$-formylhydroxylamine compounds were designed, optimized with the AutoDock 4.0.1 to investigate the interactions between the target compounds and the amino acid residues of the Escherichia coli $\mathrm{PDF} \bullet \mathrm{Ni}$ enzyme, and then synthesized through multi-step sequence starting from diethyl malonate. The structures of the compounds were characterized on the basis of spectral (FT-IR, 1H NMR and mass) analysis. All the synthesized compounds have been screened for their antimicrobial activities. It was found that the compounds 11c, 11d, 11f and $\mathbf{1 1 g}$ exhibited potent inhibitory activity against $S$. aureus in vitro.
\end{abstract}

Keywords: Peptide deformylase, inhibitors, N-formylhydroxylamine compounds, antibacterial activity

\section{Introduction}

In recent years, with the changing of environment, bacterial infections emerged rapidly and have become a serious threat to respiratory and skin infections. ${ }^{1-2}$ There is, therefore, an urgent need to identify new antibiotics to combat infectious diseases. One of the new targets receiving widespread interest from both academic and industrial researcher groups is Peptide deformylase (PDF), ${ }^{3-5}$ which is an iron containing metalloenzyme responsible for the removal of the $N$-formyl 
group from the terminal methionine residue following protein synthesis in bacteria, ${ }^{6}$ and this enzyme is a high priority target for antibiotic design. ${ }^{7}$ There are several kinds of compounds which have inhibitory activity against PDF as reported. ${ }^{8-9}$ The $N$-formyl hydroxylamine BB-3497 is an effective inhibitor $\left(\mathrm{IC}_{50}=7 \mathrm{nM}\right)$ of the Escherichia coli PDF $\bullet \mathrm{Ni}$ enzyme, exhibiting potent antibacterial activity both in vitro and in vivo. ${ }^{10}$

In order to reduce the peptide structural features to find more active compounds, we referred to the structure of the BB-3497, which was reported in the literature, ${ }^{11}$ and designed a new series of $\mathrm{N}$-formylhydroxylamine derivatives. Our intention is to synthesize new active compounds and detect their antibacterial activities by introducing one benzimidazole ring ${ }^{12-13}$ to serve as a hydrogen bond donor or accepter. At the meantime, in order to investigate the interactions between our designed compounds and the amino acid residues of the Escherichia coli PDF•Ni enzyme, a molecular docking study was also performed. After the optimization of the designed compounds, they were synthesized with our own designed multi-step reaction route and their antibiotic activities were tested in vitro also.

\section{Results and Discussion}

\section{Molecular docking study}

The molecular docking study was performed using the AutoDock 4.0.1 software. ${ }^{14}$ The crystallographic structure of Escherichia coli PDF $\bullet \mathrm{Ni}$ enzyme which is retrieved from the RSCB Protein Data Bank (PDB code 1G2A) serves as docking receptor, ${ }^{15}$ and all the synthesized compounds are selected as ligand molecules. The 50 docking runs for each ligand were clustered on the basis of root mean square deviation ( $\mathrm{RMSD}=1.0 \AA$ ) between the Cartesian coordinates of the ligand atoms and were ranked according to the binding free energy. The structure with the lowest binding free energy and the most cluster members was chosen for the optimum docking conformation.

Docking results show that all the designed molecules have similar orientations in the binding pocket of PDF enzyme, except that the terminal substituents on the benzimidazole ring have relatively large conformational differences because of their diversity on atomic composition and chemical property. The functional group $\mathrm{HCO}-\mathrm{NOH}$ in all molecules are chelated to $\mathrm{Ni}^{2+}$ ion and can effectively form hydrogen bonds with Gln50, Leu91 and Glu133, which is very similar to inhibitor BB-3497. ${ }^{16}$ The binding modes of BB-3497 and the synthesized compound 11c bound to active site of PDF $\bullet \mathrm{Ni}$ enzyme are shown in Figure 1. It should be noted that on the benzimidazole portion of 11c, the $\mathrm{N}^{3}$ atom is hydrogen bonded to the amine hydrogen of Gly89 and the bonding length is $1.96 \AA$, and another hydrogen bond which located at the $\mathrm{O}$ atom of methoxyl and residue Arg97 also forms, with a bond length of $1.84 \AA$. These strong hydrogen-bonding interactions are concomitant with the introduction of the benzimidazole ring, which means that this portion can increase the binding affinity between the target molecule and the Escherichia coli PDF• Ni 
enzyme.
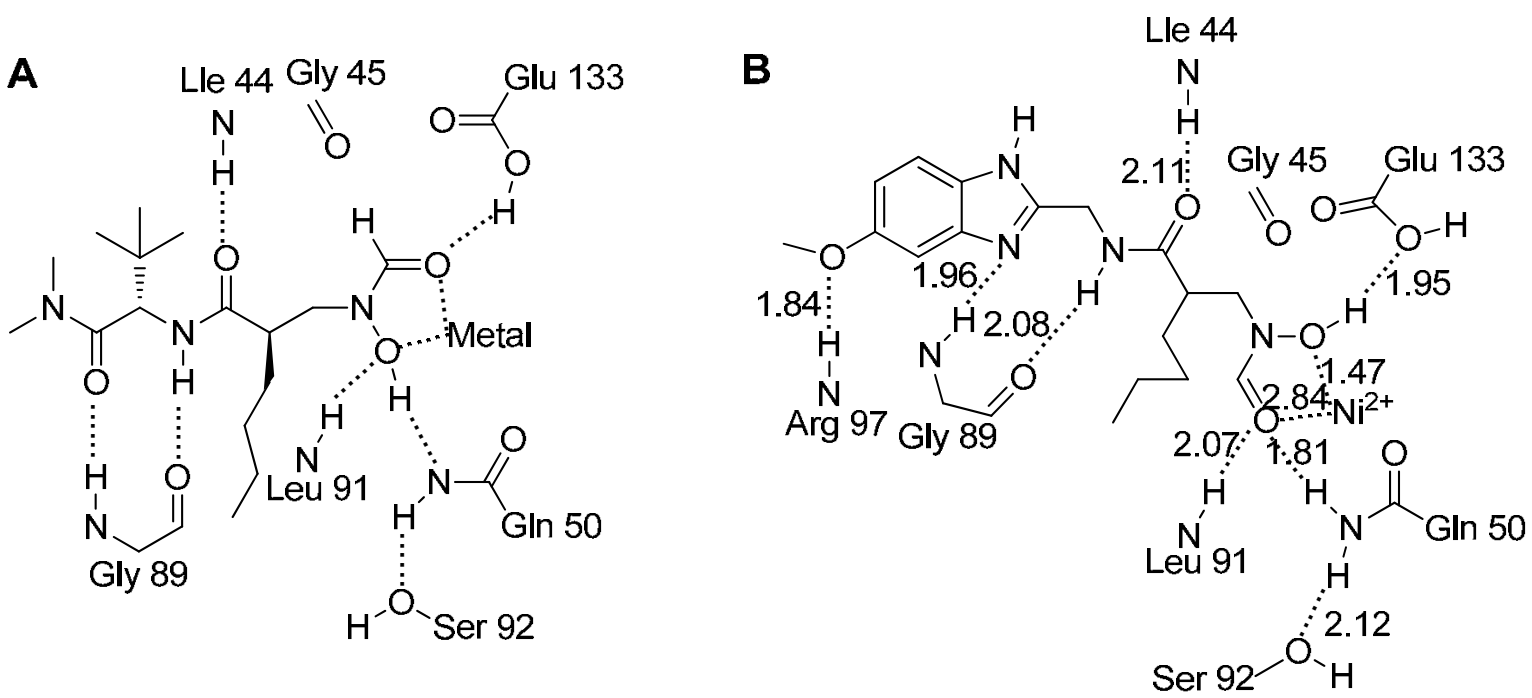

Figure 1. The binding conformation of inhibitor BB-3497 ${ }^{16}$ and 11c with E. coli PDF, shown in part A and B respectively.

\section{Chemistry}

The target compounds were prepared using the reaction sequence as shown in Scheme 1, 2. The chemical structures of the synthesized compounds were confirmed by means of their IR, ${ }^{1} \mathrm{H}$ NMR and mass spectral analysis.

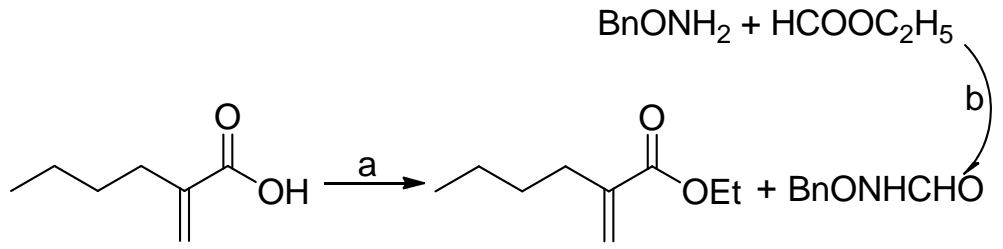

1

2

3<smiles>CCCCCN(COCCOC(=O)C(Br)CN(CCCC)COCC)CC(Br)C(=O)O</smiles>

4

5

Scheme 1. Syntheses of compounds 1-5. Reagents and conditions: (a) EtOH, $98 \% \mathrm{H}_{2} \mathrm{SO}_{4}$, reflux; (b) $\mathrm{KOH}, \mathrm{MeOH}$, rt; (c) $\mathrm{DBU}$, acetonitrile, $\mathrm{N}_{2}$, reflux; (d) $\mathrm{LiOH} \cdot \mathrm{H}_{2} \mathrm{O}, \mathrm{MeOH} / \mathrm{H}_{2} \mathrm{O}$, rt. 


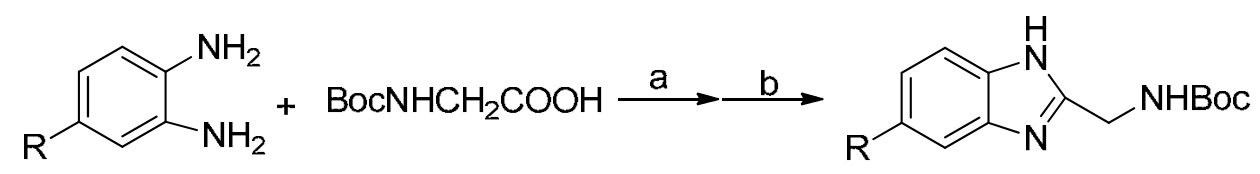

6a-g

7a-g

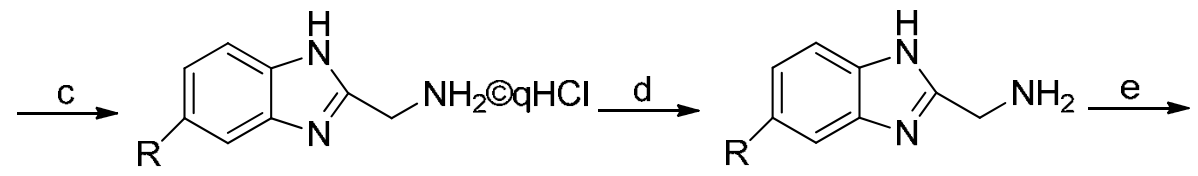

8a-g

9a-g<smiles>[R]c1ccc2[nH]c(CNC(=O)C(CCC)CN(O)C=O)nc2c1</smiles>

a: $\mathrm{R}=\mathrm{H} ; \mathrm{b}: \mathrm{R}=\mathrm{CH}_{3} ; \mathrm{c}: \mathrm{R}=\mathrm{OCH}_{3}$; $\mathrm{d}: \mathrm{R}=\mathrm{OC}_{2} \mathrm{H}_{5} ; \mathrm{e}: \mathrm{R}=\mathrm{CN}$; $: \mathrm{R}=\mathrm{COOCH}_{3} ; \mathrm{g}: \mathrm{R}=\mathrm{Cl}$

Scheme 2. Syntheses of compounds 11a-g. Reagents and conditions: (a) DCC, THF, rt; (b) HOAc, $72{ }^{\circ} \mathrm{C}$; (c) $\mathrm{HCl}, \mathrm{MeOH}, \mathrm{rt}$; (d) $\mathrm{Et}_{3} \mathrm{~N}, \mathrm{CH}_{2} \mathrm{Cl}_{2}$; (e) 5, CDMT, $\mathrm{NMM}, \mathrm{CH}_{2} \mathrm{Cl}_{2}$, rt; (f) $\mathrm{H}_{2}, 10 \% \mathrm{Pd} / \mathrm{C}$, $\mathrm{EtOH}, \mathrm{rt}$.

\section{Antibacterial activity}

All the synthesized compounds were evaluated for their antimicrobial activities. The results are summarized in Table 1. All the target compounds exhibit weak inhibitory activity against $K$. pneumonia. Compounds 11a, 11b and 11e show weak to moderate antibacterial effect against $S$. aureus, while compounds 11c, 11d, $11 \mathrm{f}$ and $\mathbf{1 1 g}$ show high activity comparable to that of the positive drug. The presence of electron-withdrawing groups on the benzene ring in generally increased the antimicrobial activity against $S$. aureus compared to compounds with electron-donating groups. Based upon the results, it is necessary to optimize the target molecule by substituting a series of electron-withdrawing groups on the benzene ring. Taken together, these results confirmed the mode of action of the target compounds and directed our design in the future. 
Table 1. The antibacterial activity of the target compounds (MIC)

\begin{tabular}{lllllc}
\hline $\begin{array}{l}\text { Sr. } \\
\text { No. }\end{array}$ & $\mathrm{R}$ & $\begin{array}{l}\text { Molecular } \\
\text { Formula }\end{array}$ & $\begin{array}{l}\text { Drug } \\
\text { Units }\end{array}$ & S. aureus & K. pneumoniae \\
\hline 11a & $\mathrm{H}$ & $\mathrm{C}_{16} \mathrm{H}_{22} \mathrm{~N}_{4} \mathrm{O}_{3}$ & $\mu \mathrm{g} / \mathrm{ml}$ & 16 & $>32$ \\
11b & $5-\mathrm{CH}_{3}$ & $\mathrm{C}_{17} \mathrm{H}_{24} \mathrm{~N}_{4} \mathrm{O}_{3}$ & $\mu \mathrm{g} / \mathrm{ml}$ & 32 & $>32$ \\
11c & $5-\mathrm{OCH}_{3}$ & $\mathrm{C}_{17} \mathrm{H}_{24} \mathrm{~N}_{4} \mathrm{O}_{4}$ & $\mu \mathrm{g} / \mathrm{ml}$ & $<1$ & $>32$ \\
$\mathbf{1 1 d}$ & $5-\mathrm{OC}_{2} \mathrm{H}_{5}$ & $\mathrm{C}_{18} \mathrm{H}_{26} \mathrm{~N}_{4} \mathrm{O}_{4}$ & $\mu \mathrm{g} / \mathrm{ml}$ & $<1$ & $>32$ \\
$\mathbf{1 1 e}$ & $5-\mathrm{CN}$ & $\mathrm{C}_{17} \mathrm{H}_{21} \mathrm{~N}_{5} \mathrm{O}_{3}$ & $\mu \mathrm{g} / \mathrm{ml}$ & 32 & $>32$ \\
$\mathbf{1 1 f}$ & $5-\mathrm{COOCH} \mathrm{H}_{3}$ & $\mathrm{C}_{18} \mathrm{H}_{24} \mathrm{~N}_{4} \mathrm{O}_{5}$ & $\mu \mathrm{g} / \mathrm{ml}$ & $<1$ & $>32$ \\
$\mathbf{1 1 g}$ & $5-\mathrm{Cl}$ & $\mathrm{C}_{16} \mathrm{H}_{21} \mathrm{ClN}_{4} \mathrm{O}_{3}$ & $\mu \mathrm{g} / \mathrm{ml}$ & $<1$ & $>32$ \\
Linezolid & & & $\mu \mathrm{g} / \mathrm{ml}$ & 1 & 1 \\
\hline
\end{tabular}

\section{Experimental Section}

General. All solvents used were of analytical grade. Melting points were determined in open capillary tubes and were uncorrected. The ${ }^{1} \mathrm{H}$ NMR spectra were recorded on a Bruker Avance DPX300 spectrometers with $\mathrm{CDCl}_{3}$ as the solvent and TMS as the internal standard. The IR spectra were measured on a Bruker Vector FTIR spectrophotometer with $\mathrm{KBr}$ pellets or film. The mass spectra were obtained with an Agilent 6510 Q-TOF spectrometer.

Synthesis of $\mathrm{N}$-(benzyloxy)formamide (3). Sodium hydroxide (5.00 g, $125.0 \mathrm{mmol})$ and ethyl formate $(23.19 \mathrm{~g}, 313.5 \mathrm{mmol})$ were added to a stirred mixture of $O$-benzylhydroxylamine $(10.00 \mathrm{~g}, 62.7 \mathrm{mmol})$ in anhydrous methanol $(120 \mathrm{ml})$, and the resulting mixture was stirred at room temperature for $16 \mathrm{~h}$. The reaction mixture was concentrated to remove methanol and then was extracted with dichloromethane $(3 \times 40 \mathrm{ml})$, and the combined extracts were washed with water, $2 \mathrm{~mol} / \mathrm{L} \mathrm{HCl}$ and brine successively and then dried over anhydrous $\mathrm{MgSO}_{4}$ followed by evaporation. The crude product was distilled under the reduced pressure to result in compound 3 in the form of a colorless oil. Yield: $70 \%$.

Synthesis of 2-methylenehexanoic acid (1). Compound 1 was prepared according to the described method. ${ }^{11}$

Synthesis of ethyl 2-methylenehexanoate (2). Compound 1 (10.00 g, $78.1 \mathrm{mmol})$ and 98\% $\mathrm{H}_{2} \mathrm{SO}_{4}(1 \mathrm{ml})$ were added to anhydrous ethanol $(50 \mathrm{ml})$, and then refluxed for $6 \mathrm{~h}$. The solvent was evaporated and the residue was basified with $\mathrm{NaHCO}_{3}$ solution. The solution was then extracted with ethyl acetate $(3 \times 30 \mathrm{ml})$ and washed with brine, and the combined extracts were dried over anhydrous $\mathrm{MgSO}_{4}$ and evaporated to afford a colorless oil. Yield: $80 \%$.

Ethyl 2-\{[N-(benzyloxy)formamido]methyl\}hexanoate (4). Compound $3(10.00 \mathrm{~g}, 66.2 \mathrm{mmol})$, compound $2(15.51 \mathrm{~g}, 99.3 \mathrm{mmol})$ and 1,8-diazabicyclo[5.4.0]undec-7-ene (DBU)(10.08 g, 
$66.2 \mathrm{mmol}$ ) were stirred in $50 \mathrm{ml}$ of acetonitrile under $\mathrm{N}_{2},{ }^{17-18}$ and then was refluxed for $8 \mathrm{~h}$. The solvent was evaporated and then the residue was extracted with ethyl acetate $(3 \times 50 \mathrm{ml})$, and the combined extracts were washed with $2 \mathrm{~mol} / \mathrm{L} \mathrm{HCl}$ and brine successively and then dried over anhydrous $\mathrm{MgSO}_{4}$ and evaporated to afford a residue, which was purified by a silica-gel column chromatography (petroleum ether / ethyl acetate / dichloromethane, 6:1:1 by volume) to yield compound 4 in the form of a colorless oil. Yield: $85 \% .{ }^{1} \mathrm{H}$ NMR $\left(300 \mathrm{MHz}, \mathrm{CDCl}_{3}, \delta \mathrm{ppm}\right)$ : 0.86-0.90 (t, 3H , $\left.J=6.9 \mathrm{~Hz}, \mathrm{CH}_{3}\right), 1.20-1.24\left(\mathrm{t}, 3 \mathrm{H}, \mathrm{CH}_{3}\right), 1.26-1.60\left(\mathrm{~m}, 6 \mathrm{H}, \mathrm{CH}_{2}\right), 2.70(\mathrm{~m}, 1 \mathrm{H}$, $\mathrm{CH}), 3.24-3.81\left(\mathrm{~m}, 2 \mathrm{H}, \mathrm{CH}_{2}\right), 4.02-4.19\left(\mathrm{~m}, 2 \mathrm{H}, \mathrm{CH}_{2}\right), 4.70-4.97\left(\mathrm{~m}, 2 \mathrm{H}, \mathrm{CH}_{2}\right), 7.38(\mathrm{~m}, 5 \mathrm{H}$, Ar-H), 7.94 (brs, 0.32H, CHO), 8.15 (brs, 0.68H, CHO).

Synthesis of $2-\{[N$-(benzyloxy)formamido]methyl\}hexanoic acid (5). Compound 4 (5.01 g, $16.3 \mathrm{mmol})$ and $\mathrm{LiOH} \cdot \mathrm{H}_{2} \mathrm{O}(0.79 \mathrm{~g}, 17.9 \mathrm{mmol})$ were stirred in $75 \mathrm{ml}$ of 2:1 (volume ratio) methanol/water at room temperature for $24 \mathrm{~h}$. The reaction mixture was concentrated to remove methanol, and the residue was diluted with water $(10 \mathrm{ml})$. The mixture was then extracted with ethyl acetate $(3 \times 20 \mathrm{ml})$, and the aqueous phase was acidified to $\mathrm{pH}<3$ with concentrated $\mathrm{HCl}$. The acidic aqueous solution was then extracted with ethyl acetate $(3 \times 30 \mathrm{ml})$, and the extracts were dried over anhydrous $\mathrm{MgSO}_{4}$ and evaporated to afford a residue, which was purified by a silica-gel column chromatography (petroleum ether / ethyl acetate / dichloromethane, 6:1:1 by volume, containing some formyl acid) to give compound 5 in the form of a light yellow oil. Yield: 75\%. ${ }^{1} \mathrm{H}$ NMR (300MHz, $\left.\mathrm{CDCl}_{3}, \delta \mathrm{ppm}\right): 0.85-0.89\left(\mathrm{t}, 3 \mathrm{H}, J=6.6 \mathrm{~Hz}, \mathrm{CH}_{3}\right), 1.30-1.61(\mathrm{~m}, 6 \mathrm{H}$, $\left.\mathrm{CH}_{2}\right), 2.74(\mathrm{~m}, 1 \mathrm{H}, \mathrm{CH}), 3.24-3.85\left(\mathrm{~m}, 2 \mathrm{H}, \mathrm{CH}_{2}\right), 4.75-4.97\left(\mathrm{~m}, 2 \mathrm{H}, \mathrm{CH}_{2}\right), 7.35-7.41(\mathrm{~m}, 5 \mathrm{H}$, Ar-H), 7.99 (brs, 0.35H, CHO), 8.16 (brs, 0.65H, CHO), 10.91 (brs, $1 \mathrm{H}, \mathrm{COOH}$ ).

\section{Synthesis of tert-butyl (1H-benzo[d]imidazol-2-yl)methylcarbamate (7a). Typical procedure}

Benzene-1, 2-diamine (1.05 g, $9.72 \mathrm{mmol})$ and $N$-(tert-butoxycarbonyl)glycine $(1.71 \mathrm{~g}$, $9.72 \mathrm{mmol}$ ) were dissolved in $30 \mathrm{ml}$ of THF and cooled to $0{ }^{\circ} \mathrm{C}$. Into the above solution was added $N, N^{\prime}$-dicyclohexylcarbodiimide $(2.41 \mathrm{~g}, 11.7 \mathrm{mmol})$ in batches and the mixture was stirred at $0{ }^{\circ} \mathrm{C}$ for half an hour and then at room temperature overnight. The reaction mixture was filtrated and evaporated to afford a brown oil, which was purified by a silica-gel column chromatography (dichloromethane / methanol, 25:1 by volume) to get a yellow solid (2.24 g). The solid was dissolved in $20 \mathrm{ml}$ of acetic acid and the solution was stirred at $72{ }^{\circ} \mathrm{C}$ for $8 \mathrm{~h}$. The acetic acid was removed under reduced pressure and the crude compound was purified by a silica-gel column chromatography (dichloromethane / methanol, 25:1 by volume) to afford a white solid. Yield: 82\%. m.p. 181-183 ${ }^{\circ} \mathrm{C}$. IR ( $\left.\mathrm{KBr}\right) \mathrm{cm}^{-1}: 3343$ (-NH-), 3058, 2980, 2940 (CH, aliphatic), 1686 $(>\mathrm{C}=\mathrm{O}), 1529$ (-C=C-), 736 (-Ar-); ${ }^{1} \mathrm{H} \mathrm{NMR}\left(300 \mathrm{MHz}, \mathrm{CDCl}_{3}, \delta \mathrm{ppm}\right): 1.45\left(\mathrm{~s}, 9 \mathrm{H}, \mathrm{CH}_{3}\right)$, 4.53-4.55 (d, 2H, J=6.3 Hz, $\mathrm{CH}_{2}$ ), 6.12 (brs, 1H, NH), 7.22-7.25 (m, 2H, Ar-H), 7.54-7.60 (m, 2H, Ar-H); HRMS (ESI): $[\mathrm{M}+\mathrm{H}]^{+}$calcd $\mathrm{m} / \mathrm{z} 248.1400$, found 248.1397.

tert-Butyl (5-methyl-1H-benzo[d]imidazol-2-yl)methylcarbamate (7b). Compound 7b was obtained as a white solid. Yield: $81 \%$. m.p. $171-172{ }^{\circ} \mathrm{C}$. IR (KBr) cm ${ }^{-1}: 3336$ (-NH-), 2988, 2911 (CH, aliphatic), $1679(>\mathrm{C}=\mathrm{O}), 1521(-\mathrm{C}=\mathrm{C}-) ;{ }^{1} \mathrm{H} \mathrm{NMR}\left(300 \mathrm{MHz}, \mathrm{CDCl}_{3}, \delta \mathrm{ppm}\right): 1.46(\mathrm{~s}, 9 \mathrm{H}$, $\left.\mathrm{CH}_{3}\right), 2.46\left(\mathrm{~s}, 3 \mathrm{H}, \mathrm{CH}_{3}\right), 4.48-4.50\left(\mathrm{~d}, 2 \mathrm{H}, J=6.3 \mathrm{~Hz}, \mathrm{CH}_{2}\right), 5.64$ (brs, 1H, NH), 7.09 (m, H, 
Ar-H), 7.34 (m, H, Ar-H), 7.45 (m, H, Ar-H); HRMS (ESI): [M + H] $]^{+}$calcd m/z 262.1556, found 262.1546.

\section{$N$-[(1H-Benzo[d]imidazol-2-yl)methyl]-2-\{[N-(benzyloxy)formamido]methyl $\}$ hexanamide (10a). Typical procedure}

Compound 7a $(1.72 \mathrm{~g}, 4.21 \mathrm{mmol})$ was added to a stirred mixture of $2 \mathrm{~mol} / \mathrm{L} \mathrm{HCl}$ in methanol $(20 \mathrm{ml})$ at room temperature for $8 \mathrm{~h}$. The reaction mixture was concentrated, and then was washed with ether to yield a white solid 8a $(1.16 \mathrm{~g})$. To a stirred solution of 4,6-dimethoxy-2-chloro-1,3,5-triazine (CDMT) (1.00 g, $5.72 \mathrm{mmol})$ and 2-[(N-hydroxyformamido)methyl]hexanoic acid $(1.45 \mathrm{~g}, 5.20 \mathrm{mmol})$ in dry $\mathrm{CH}_{2} \mathrm{Cl}_{2}(30 \mathrm{ml})$. $\mathrm{N}$-methylmorpholine (NMM, $683 \mathrm{mg}, 6.76 \mathrm{mmol}$ ) was added dropwise at $0{ }^{\circ} \mathrm{C}$ and was continuously stirred at $0{ }^{\circ} \mathrm{C}$ for $4 \mathrm{~h}$. To the crude solution of $(1 \mathrm{H}$-benzo[d]imidazol-2-yl) methanamine hydrochloride $(764 \mathrm{mg}, 5.20 \mathrm{mmol})$ was added triethylamine $(526 \mathrm{mg}, 5.20 \mathrm{mmol})$ in $\mathrm{CH}_{2} \mathrm{Cl}_{2}(5 \mathrm{ml})$ at $0{ }^{\circ} \mathrm{C}$. Stirring was continued for $2 \mathrm{~h}$ and then the mixture was left overnight at room temperature. The solvent was evaporated and the residue was diluted with $\mathrm{CH}_{2} \mathrm{Cl}_{2}$. The suspension was washed successively with $\mathrm{H}_{2} \mathrm{O}, 0.5 \mathrm{~mol} / \mathrm{L} \mathrm{HCl}$, saturated $\mathrm{NaHCO}_{3}$, and brine. The organic phase was dried and evaporated to give a white solid. The crude product was purified by a silica-gel column chromatography (dichloromethane / methanol, 30:1 by volume) to get a white solid. Yield: 89\%. m.p. $114-117{ }^{\circ} \mathrm{C}$. IR (KBr) cm${ }^{-1}: 3298$ (-NH-), 2958, 2930 (CH, aliphatic), $1679(>\mathrm{C}=\mathrm{O}), 1554$ (-C=C-), 1384 (-CHO), 748 (-Ar-); ${ }^{1} \mathrm{H}$ NMR (300 MHz, CDCl 3 , $\delta$ ppm): 0.80-0.84 (t, 3H, J=6.9 Hz, $\mathrm{CH}_{3}$ ), 1.25-1.63 (brm, 6H, $\left.\mathrm{CH}_{2}\right), 2.64$ (m, 1H, CH), 3.20-3.90 (brm, $2 \mathrm{H}, \mathrm{CH}_{2}$ ), 4.36-4.42 (dd, $\left.1 \mathrm{H}, J=4.5,4.5 \mathrm{~Hz}, \mathrm{CH}_{2}\right), 4.71$ (brm, 3H, $\mathrm{CH}_{2}$ ), 7.21-7.24 (m, 2H, Ar-H), 7.26-7.30 (m, 5H, Ar-H), 7.51 (m, 2H, Ar-H), 7.96 (brs, 1H, CHO); HRMS (ESI): [M + $\mathrm{H}]^{+}$calcd $m / z$ 409.2240, found 409.2244.

2-(( $N$-(Benzyloxy)formamido)methyl)- $N$-((5-methyl-1H-benzo[d]imidazol-2-yl)methyl)hexa namide(10b). Compound 10b was obtained as a white solid. Yield: 82\%. m.p. $60-62{ }^{\circ} \mathrm{C}$. IR (KBr) $\mathrm{m}^{-1}: 3277$ (-NH-), 2948, 2930 (CH, aliphatic), 1677 (>C=O), $1546(-\mathrm{C}=\mathrm{C}-), 1382(-\mathrm{CHO}) ;{ }^{1} \mathrm{H}$ NMR (300 MHz, $\left.\mathrm{CDCl}_{3}, \delta \mathrm{ppm}\right): 0.79-0.84\left(\mathrm{t}, 3 \mathrm{H}, J=7.2 \mathrm{~Hz}, \mathrm{CH}_{3}\right), 1.24-1.63\left(\mathrm{~m}, 6 \mathrm{H}, \mathrm{CH}_{2}\right)$, $2.44\left(\mathrm{~s}, 3 \mathrm{H}, \mathrm{CH}_{3}\right), 2.66(\mathrm{~m}, 1 \mathrm{H}, \mathrm{CH}), 3.17-3.88\left(\mathrm{~m}, 2 \mathrm{H}, \mathrm{CH}_{2}\right), 4.31-4.37$ (dd, $1 \mathrm{H}, J=4.8,4.8 \mathrm{~Hz}$, $\left.\mathrm{CH}_{2}\right)$, 4.61-4.69 (m, 3H, $\left.\mathrm{CH}_{2}\right), 7.02-7.05(\mathrm{~d}, 1 \mathrm{H}, \mathrm{J}=8.1 \mathrm{~Hz}, \mathrm{Ar}-\mathrm{H}), 7.30$ (m, 5H, Ar-H), 7.39 (m, 1H, Ar-H), 7.75 (m, 1H, Ar-H ), 7.95 (brs, 1H, CHO); HRMS (ESI): [M+ H] $]^{+}$calcd m/z 423.2397, found 423.2390 .

\section{2-[( $N$-Hydroxyformamido)methyl]- $N$-[(5-substituted-1H-benzo[d]imidazol-2-yl)methyl]hex}

\section{anamide (11a-g). General procedure}

$10 \% \mathrm{Pd} / \mathrm{C}(0.20 \mathrm{~g}$, containing $65.8 \%$ water $)$ was added to a stirred mixture of compound 10a $(0.80 \mathrm{~g}, 1.96 \mathrm{mmol})$ and anhydrous ethanol $(20 \mathrm{ml})$ under $\mathrm{H}_{2}$ at room temperature for $3 \mathrm{~h}$. The filtrate was evaporated to yield a white solid, which was purified by a silica-gel column chromatography (dichloromethane / methanol, 20:1 by volume). 
$N$-[(1H-Benzo[d]imidazol-2-yl)methyl]-2-[( $N$-hydroxyformamido)methyl]hexanamide (11a). Compound 11a was obtained as a white solid. Yield: $92 \%$. m.p. $85-86{ }^{\circ} \mathrm{C}$. IR $(\mathrm{KBr}) \mathrm{cm}^{-1}: 3279$ (-NH-), 2956, 2930 (CH, aliphatic), 1655 (>C=O), 1540 (-C=C-), 1384 (-CHO), 743 (-Ar-); ${ }^{1} \mathrm{H}$ NMR (300 MHz, DMSO- $d_{6}, \delta$ ppm): 0.82-0.83 (t, $\left.3 \mathrm{H}, \mathrm{CH}_{3}\right), 1.23-1.44\left(\mathrm{~m}, 6 \mathrm{H}, \mathrm{CH}_{2}\right), 2.64-2.73$ (m, $1 \mathrm{H}, \mathrm{CH}), 3.39-3.80\left(\mathrm{~m}, 2 \mathrm{H}, \mathrm{CH}_{2}\right)$, 4.29-4.76 (m, 2H, $\left.\mathrm{CH}_{2}\right), 7.14-7.19(\mathrm{~m}, 2 \mathrm{H}, \mathrm{Ar}-\mathrm{H}), 7.51(\mathrm{~m}$, $2 \mathrm{H}, \mathrm{Ar}-\mathrm{H}), 7.87$ (brs, $0.49 \mathrm{H}, \mathrm{CHO}), 8.30$ (brs, $0.51 \mathrm{H}, \mathrm{CHO}) ;{ }^{13} \mathrm{C}$ NMR $\left(75 \mathrm{MHz}, \mathrm{DMSO}-d_{6}, \delta\right.$ ppm): 14.34, 22.61, 22.67, 29.14, 29.28, 29.55, 36.97, 37.27, 44.10, 44.39, 49.76, 52.13, 122.11, 152.62, 153.23, 157.97, 162.46, 173.62, 173.84; HRMS (ESI): $[\mathrm{M}+\mathrm{H}]^{+}$calcd $\mathrm{m} / \mathrm{z} 319.1771$, found 319.1761 .

2-[( $N$-Hydroxyformamido)methyl]- $N$-[(5-methyl-1H-benzo[d]imidazol-2-yl)methyl]hexana mide (11b). Compound 11b was obtained as a white solid. Yield: $91 \%$. m.p. $93-95{ }^{\circ} \mathrm{C}$. IR $(\mathrm{KBr})$ $\mathrm{cm}^{-1}: 3271$ (-NH-), 2957, 2929 (CH, aliphatic), $1656(>\mathrm{C}=\mathrm{O}), 1543(-\mathrm{C}=\mathrm{C}-), 1384(-\mathrm{CHO}) ;{ }^{1} \mathrm{H}$ NMR (300 MHz, DMSO- $d_{6}, \delta$ ppm): 0.82-0.84 (t, 3H, $\left.\mathrm{CH}_{3}\right), 1.23-1.48\left(\mathrm{~m}, 6 \mathrm{H}, \mathrm{CH}_{2}\right), 2.39(\mathrm{~s}, 3 \mathrm{H}$, $\left.\mathrm{CH}_{3}\right), 2.61-2.72(\mathrm{~m}, 1 \mathrm{H}, \mathrm{CH}), 3.38-3.80\left(\mathrm{~m}, 2 \mathrm{H}, \mathrm{CH}_{2}\right), 4.24-4.76\left(\mathrm{~m}, 2 \mathrm{H}, \mathrm{CH}_{2}\right), 6.96-7.01(\mathrm{t}, 1 \mathrm{H}$, $J=8.1 \mathrm{~Hz}$, Ar-H), 7.29 (s, $1 \mathrm{H}$, Ar-H), 7.36-7.39 (d, $1 \mathrm{H}, J=8.1 \mathrm{~Hz}, \mathrm{Ar}-\mathrm{H}), 7.87$ (brs, 0.49H, CHO), 8.30 (brs, $0.51 \mathrm{H}, \mathrm{CHO}$ ); ${ }^{13} \mathrm{C}$ NMR (75 MHz, DMSO-d 6 , $\delta$ ppm): 14.33, 21.69, 22.59, $22.65,29.15,29.29,29.53,36.90,37.23,44.21,44.47,49.88,52.18,123.47,123.75,152.25$, 152.89, 157.97, 162.44, 173.59, 173.82; HRMS (ESI): $[\mathrm{M}+\mathrm{H}]^{+}$calcd $\mathrm{m} / \mathrm{z} 333.1927$, found 333.1931 .

2-[( $N$-hydroxyformamido)methyl]- $N$-[(5-methoxy- $1 H$-benzo[d]imidazol-2-yl)methyl]hexana mide (11c). Compound 11c was obtained as a white solid. Yield: $89 \%$. m.p. $92-94{ }^{\circ} \mathrm{C}$. IR $(\mathrm{KBr})$ $\mathrm{cm}^{-1}$ : 3282 (-NH-), 2956, 2930 (CH, aliphatic), 1655 (>C =O), 1541 (-C=C-), 1384 (-CHO), 1031 (-C-O-C-); ${ }^{1} \mathrm{H}$ NMR (300 MHz, DMSO- $\left.d_{6}, \delta \mathrm{ppm}\right): 0.84$ (t, $\left.3 \mathrm{H}, \mathrm{CH}_{3}\right), 1.23-1.47\left(\mathrm{~m}, 6 \mathrm{H}, \mathrm{CH}_{2}\right.$ ), 2.58-2.72 (m, 1H, CH), 3.39-3.77 (m, 4H, $\left.\mathrm{CH}_{2}\right), 4.24-4.73\left(\mathrm{~m}, 2 \mathrm{H}, \mathrm{CH}_{2}\right), 6.77-6.81(\mathrm{t}, 1 \mathrm{H}, J=6.3$ $\mathrm{Hz}, \mathrm{Ar}-\mathrm{H}), 6.99$ (s, 1H, Ar-H), 7.37-7.40 (d, 1H, J=8.7 Hz, Ar-H), 7.87 (brs, 0.50H, CHO), 8.30 (brs, 0.50H, CHO); HRMS (ESI): $[\mathrm{M}+\mathrm{H}]^{+}$calcd $\mathrm{m} / \mathrm{z} 349.1877$, found 349.1861 . $N$-[(5-ethoxy-1H-benzo[d]imidazol-2-yl)methyl]-2-[( $N$-hydroxyformamido)methyl]hexanam ide (11d). Compound 11d was obtained as a white solid. Yield: $85 \%$. m.p. 80-83 ${ }^{\circ} \mathrm{C}$. IR ( $\left.\mathrm{KBr}\right)$ $\mathrm{cm}^{-1}$ : 3277 (-NH-), 2957, 2929 (CH, aliphatic), 1655 ( $\left.>\mathrm{C}=\mathrm{O}\right), 1541$ (-C=C-), 1384 (-CHO), 1044 (-C-O-C-); ${ }^{1} \mathrm{H}$ NMR $\left(300 \mathrm{MHz}, \mathrm{DMSO}-d_{6}, \delta \mathrm{ppm}\right): 0.84\left(\mathrm{t}, 3 \mathrm{H}, \mathrm{CH}_{3}\right), 1.23\left(\mathrm{~m}, 5 \mathrm{H}, \mathrm{CH}_{2}\right)$, 1.31-1.36 (t, 3H, J=6.9 Hz, $\left.\mathrm{CH}_{3}\right), 1.44\left(\mathrm{~m}, 1 \mathrm{H}, \mathrm{CH}_{2}\right), 2.62-2.72(\mathrm{~m}, 1 \mathrm{H}, \mathrm{CH}), 3.39-3.79(\mathrm{~m}, 2 \mathrm{H}$, $\left.\mathrm{CH}_{2}\right), 4.00-4.02\left(\mathrm{~m}, 2 \mathrm{H}, \mathrm{CH}_{2}\right), 4.24-4.73\left(\mathrm{~m}, 2 \mathrm{H}, \mathrm{CH}_{2}\right), 6.78(\mathrm{~s}, 1 \mathrm{H}, \mathrm{Ar}-\mathrm{H}), 6.98(\mathrm{~s}, 1 \mathrm{H}, \mathrm{Ar}-\mathrm{H})$, 7.36-7.39 (d, $1 \mathrm{H}, J=8.1 \mathrm{~Hz}, \mathrm{Ar}-\mathrm{H}$ ), 7.87 (brs, 0.49H, CHO), 8.30 (brs, 0.51H, CHO); HRMS (ESI): $[\mathrm{M}+\mathrm{H}]^{+}$calcd $m / z$ 363.2033, found 363.2039.

$N$-[(5-Cyano-1H-benzo[d]imidazol-2-yl)methyl]-2-[( $N$-hydroxyformamido)methyl)hexanam ide (11e). Compound 11e was obtained as a white solid. Yield: $86 \%$. m.p. $74-77{ }^{\circ} \mathrm{C}$. IR ( $\mathrm{KBr}$ ) $\mathrm{cm}^{-1}$ : 3281 (-NH-), 2953, 2929 (CH, aliphatic), $2224(-\mathrm{CN}), 1655(>\mathrm{C}=\mathrm{O}), 1539$ (-C=C-), 1384 (-CHO); ${ }^{1} \mathrm{H}$ NMR $\left(300 \mathrm{MHz}\right.$, DMSO- $\left.d_{6}, \delta \mathrm{ppm}\right): 0.79-0.83$ (t, $\left.3 \mathrm{H}, J=6.9 \mathrm{~Hz}, \mathrm{CH}_{3}\right), 1.21-1.38$ (m, $\left.6 \mathrm{H}, \mathrm{CH}_{2}\right), 2.66-2.74(\mathrm{~m}, 1 \mathrm{H}, \mathrm{CH}), 3.38-3.77\left(\mathrm{~m}, 2 \mathrm{H}, \mathrm{CH}_{2}\right), 4.41-4.67\left(\mathrm{~m}, 2 \mathrm{H}, \mathrm{CH}_{2}\right), 7.55-7.58(\mathrm{~d}$, $1 \mathrm{H}, J=8.1 \mathrm{~Hz}, \mathrm{Ar}-\mathrm{H}), 7.66-7.69$ (d, $1 \mathrm{H}, J=8.4 \mathrm{~Hz}, \mathrm{Ar}-\mathrm{H}), 8.05$ (s, 1H, Ar-H), 7.86 (brs, $0.51 \mathrm{H}$, 
CHO), 8.30 (brs, 0.49H, CHO); ${ }^{13} \mathrm{C}$ NMR (75 MHz, DMSO- $d_{6}, \delta$ ppm): 14.32, 22.64, 29.08, 29.60, 37.30, 37.42, 43.77, 43.97, 49.08, 51.97, 104.00, 104.16, 120.48, 125.74, 125.86, 156.20, 156.53, 157.89, 162.55, 173.80, 173.93; HRMS (ESI): $[\mathrm{M}+\mathrm{H}]^{+}$calcd $\mathrm{m} / \mathrm{z} 344.1723$, found 344.1728 .

Methyl 2-\{\{2-[( $N$-hydroxyformamido)methyl]hexanamido\}methyl $\} \quad \mathbf{H}$-benzo[d] imidazole-5-carboxylate (11f). Compound 11f was obtained as a white solid. Yield: 84\%. m.p. 86-88 ${ }^{\circ} \mathrm{C}$. IR (KBr) cm ${ }^{-1}: 3287$ (-NH-), 2955, 2930 (CH, aliphatic), 1718, $1659(>\mathrm{C}=\mathrm{O}), 1541$ $(-\mathrm{C}=\mathrm{C}-), 1384(-\mathrm{CHO}) ;{ }^{1} \mathrm{H}$ NMR $\left(300 \mathrm{MHz}, \mathrm{DMSO}-d_{6}, \delta \mathrm{ppm}\right): 0.80-0.82\left(\mathrm{t}, 3 \mathrm{H}, \mathrm{CH}_{3}\right), 1.22-1.44$ $\left(\mathrm{m}, 6 \mathrm{H}, \mathrm{CH}_{2}\right), 2.66-2.74(\mathrm{~m}, 1 \mathrm{H}, \mathrm{CH}), 3.41-3.80\left(\mathrm{~m}, 2 \mathrm{H}, \mathrm{CH}_{2}\right), 3.86\left(\mathrm{~s}, 3 \mathrm{H}, \mathrm{CH}_{3}\right), 4.38-4.71(\mathrm{~m}$, $\left.2 \mathrm{H}, \mathrm{CH}_{2}\right), 7.59-7.61(\mathrm{~d}, 1 \mathrm{H}, J=7.8 \mathrm{~Hz}, \mathrm{Ar}-\mathrm{H}), 7.82-7.84(\mathrm{~d}, 1 \mathrm{H}, J=8.1 \mathrm{~Hz}, \mathrm{Ar}-\mathrm{H}), 8.12(\mathrm{~s}, 1 \mathrm{H}$, Ar-H), 7.87 (brs, 0.55H, CHO), 8.31 (brs, 0.45H, CHO); ${ }^{13} \mathrm{C}$ NMR (75 MHz, DMSO- $d_{6}, \delta$ ppm): 14.32, 22.64, 29.12, 29.21, 29.59, 37.22, 37.35, 43.98, 44.16, 49.44, 52.06, 52.44, 111.88, 113.60, $118.69,120.49,123.71,138.33,142.82,146.72,155.11,156.09,157.95,162.48,167.19,167.25$, 173.75, 173.93; HRMS (ESI): $[\mathrm{M}+\mathrm{H}]^{+}$calcd $\mathrm{m} / \mathrm{z}$ 377.1826, found 377.1825 .

$\boldsymbol{N}$-[(5-Chloro-1H-benzo[d]imidazol-2-yl)methyl]-2-[( $\boldsymbol{N}$-hydroxyformamido)methyl]hexana mide (11g). Compound 11g was obtained as a white solid. Yield: 79\%. m.p. 96-98 ${ }^{\circ} \mathrm{C}$. IR $(\mathrm{KBr})$ $\mathrm{cm}^{-1}$ : 3276 (-NH-), 2957, 2930 (CH, aliphatic), 1656 (>C=O), 1541 (-C=C-), $706(-\mathrm{C}-\mathrm{Cl}-) ;{ }^{1} \mathrm{H}$ NMR (300 MHz, DMSO-d $d_{6} \delta$ ppm): 0.79-0.82 (t, 3H, $\left.\mathrm{CH}_{3}\right), 1.22-1.43\left(\mathrm{~m}, 6 \mathrm{H}, \mathrm{CH}_{2}\right), 2.64-2.73$ (m, 1H, CH), 3.39-3.78 (m, 2H, $\left.\mathrm{CH}_{2}\right), 4.33-4.68\left(\mathrm{~m}, 2 \mathrm{H}, \mathrm{CH}_{2}\right), 7.19(\mathrm{~s}, 1 \mathrm{H}, \mathrm{Ar}-\mathrm{H}), 7.50-7.56(\mathrm{~m}$, $2 \mathrm{H}, \mathrm{Ar}-\mathrm{H}), 7.86$ (brs, $0.51 \mathrm{H}, \mathrm{CHO}), 8.30$ (brs, 0.49H, CHO); HRMS (ESI): $\left[\mathrm{M}\left({ }^{35} \mathrm{Cl}\right)+\mathrm{H}\right]^{+}$calcd $\mathrm{m} / \mathrm{z}$ 353.1381, found 353.1380 .

\section{Antibacterial activity}

The compounds 11a-g were screened in vitro for their antibacterial activity against $S$. aureus and K. pneumoniae using Linezolid as standard and DMSO as solvent control. The method of micro-dilution was performed using Muller-Hinton agar (Hi-medium) to test the seven new compounds of their inhibitory activity. After $18 \mathrm{~h}$ of incubation at $37{ }^{\circ} \mathrm{C}$, minimum inhibitory concentration (MIC) of the synthesized compounds were observed and shown in Table 1.

\section{Acknowledgements}

The authors would like to thank the National Basic Research Priority Program (2003CCA027) and the Scientific Technological Project in Shandong Province (2009GG20002027) for financial support. 


\section{References}

1. Tomasz, A. N. Engl. J. Med. 1994, 330, 1247.

2. Boularot, A.; Giglione, C.; Petit, S.; Duroc, Y.; Sousa, R. A.; Larue, V.; Cresteil, T.; Dardel, F.; Artaud, I.; Meinnel, T. J. Med. Chem. 2007, 50, 10.

3. (a) Pei, D. Emerg. Ther. Targets 2001, 5, 23. (b) Giglione, C.; Meinnel, T. Emerg. Ther. Targets 2001, 5, 41. (c) Yuan, Z.; Trias, J.; White, R. J. Drug Discov. Today 2001, 6, 955. (d) Clements, J. M.; Ayscough, A. P.; Keavey, K.; East, S. P. Curr. Med. Chem.-Anti-Infect. Agents 2002, 1, 239.

4. Leeds, J. A.; Dean, C. R. Curr. Opin. Pharmacol. 2006, 6, 445.

5. Yuan, Z.; White, R. J. Biochem. Pharmacol. 2006, 71, 1042.

6. (a) Adams, J. M.; Capecchi, M. Proc. Natl. Acad. Sci. U.S.A. 1966, 55, 147. (b) Adams, J. M. J. Mol. Biol. 1968, 33, 571.

7. Berg, A. K.; Manokaran, S.; Eiler, D.; Kooren, J.; Mallik, S.; Srivastava, D. K. Protein Sci. 2007, 17, 11.

8. Giglione, C.; Pierre, M.; Meinnel, T. Mol Microbiol. 2000, 36, 1197.

9. Butler, M. S.; Buss, A. D. Biochem. Pharmacol. 2006, 71, 919.

10. Davies, S. J.; Ayscough, A. P.; Beckett, R. P.; Clements, J. M.; Doel, S.; Pratt, L. M.; Spavold, Z. M.; Thomas, S. W.; Whittaker, M. Bioorg. Med. Chem. Lett 2003, 13, 2715.

11. Hunter, M. G.; Beckett, R. P.; Clements, J. M.; Whittaker, M.; Davies, S. J.; Pratt, L. M.; Spavold, Z. M.; Launchbury, S. U.S. Patent 6423 690, 2002.

12. Arya, P.; Rao, N. V.; Singkhonrat, J. J. Org. Chem. 2000, 65, 1881.

13. Natchus, M. G.; Cheng, M.; Wahl, C. T.; Pikul, S.; Almsted, N. G.; Bradley, R. S.; Taiwo, Y. O.; Mieling, G. E.; Dunaway, C. M.; Snider, C. E.; Mclver, J. M.; Barnett, B. L.; Mcphail, S. J.; Anastasio, M. B.; De, B. Bioorganic Med. Chem. Lett. 1998, 8, 2077.

14. Web address: http://www.scripps.edu/mb/olson/doc/autodock/.

15. RSCB Protein Data Bank, web address: http://www.rscb.org/pdb/.

16. Clements, J. M.; Beckett, R. P.; Brown, A.; Catlin, G.; Lobell, M.; Palan, S.; Thomas, W.; Whittaker, M.; Wood, S.; Salama, S.; Baker, P. J.; Rodgers, H. F.; Barynin, V.; Rice, D. W.; Hunter, M. G. Antimicrob Agents Ch. 2001, 45, 563.

17. Gimbert, C.; Moreno-Manas, M.; Perez, E.; Vallribera, A. Tetrahedron 2007, 63, 8305.

18. Price, S.; Osbourn, S. E. Org. Lett. 2005, 7, 3761. 